\title{
Improved classes of chain type estimators for ratio of two population means using two auxiliary characters in the presence of non-response
}

\author{
B. B. Khare ${ }^{1 *}$, Kamlesh Kumar ${ }^{1}$, Usha Srivastava ${ }^{2}$ \\ ${ }^{1}$ Department of Statistics, Banaras Hindu University, Varanasi, India \\ ${ }^{2}$ Statistics Section, M.M.V., Banaras Hindu University, Varanasi, India \\ ${ }^{I}$ Department of Statistics, Banaras Hindu University, Varanasi, India \\ *Corresponding author E-mail: bbkhare56@yahoo.com
}

\begin{abstract}
In the case when the population mean of auxiliary character is not known but population mean of another additional auxiliary character is known, improved classes of chain type estimators for ratio of two population means of study characters in the presence of non-response have been proposed and their properties are studied. The proposed classes of chain type estimators have been found to be more efficient than the corresponding estimators in case of fixed sample sizes and also in case of the fixed cost. It is also found that the proposed classes of chain type estimators have less total cost in comparison to the cost incurred for the corresponding estimators in case of the specified variance. An empirical study has been given in support of the problem under investigation.
\end{abstract}

Keywords: Auxiliary characters, Bias, Mean square error, Non-response, Two phase sampling

\section{Introduction}

The information on auxiliary characters plays a significant role for the estimation of ratio of two population means in the field of agricultural, socio-economic, forest surveys and medical science. For example, a health researcher may be interested in estimating the growth index by measuring the ratio of weight to height using chest circumference and skull circumference as auxiliary characters.

The problem of non-response generally occurs due to failure to measure some units selected in the sample. Hansen and Hurwitz [1] first gave a technique of sub-sampling from the non-respondents to deal with the problem of non-response in mail surveys. Using Hansen and Hurwitz [1] technique, Khare and Pandey [2], Khare and Sinha [3,4,5] have proposed different estimators for the estimation of ratio of two population means in case of known and unknown population means of auxiliary characters in the presence of non-response.

In the case when the population mean of auxiliary character is not known but population mean of another additional auxiliary character is known, which may be cheaper and less correlated to the study character in comparison to the main auxiliary character. In this situation, the estimators for the estimation of ratio of two population means were first considered by Srivastava et al. [6, 7] and Khare and Srivastava [8]. Further, general class of estimators for estimation of ratio of two populations means were proposed by Singh et al. [9] and Ahmad [10]. In such situation, Khare et al. [11] have proposed chain type estimators for estimation of ratio of two population means in the presence of non-response. In this paper, improved classes of chain type estimators for the estimation of ratio of two population means using two auxiliary characters in the presence of non-response have been proposed. The expressions for bias and mean square errors of the proposed classes of chain type estimators are obtained. The optimum values of first phase sample $\left(n^{\prime}\right)$, second phase sample $(n)$ and the sub-sampling fraction $\left(k^{-1}\right)$ of non-responding part have been determined for the fixed cost and also for the specified variance. An empirical study has also been supported to show the performance of the estimators. 


\section{The estimators}

Let $\overline{Y_{i}}(i=1,2), \bar{X}$ and $\bar{Z}$ denote the population means of study characters $y_{i}(i=1,2)$, auxiliary $x$ and additional characters $z$ having $j-t$ th values $Y_{i j}, X_{j}$ and $Z_{j}, j=1,2, \ldots, N$. Suppose the population of size $N$ is divided in $N_{1}$ responding and $N_{2}$ non-responding units. Now, a sample of size $n(<N)$ is drawn from population of size $N$ by using simple random sampling without replacement (SRSWOR) method of sampling and it is observed that $n_{1}$ units respond and $n_{2}$ units do not respond in the sample of size $n$ for the study characters $y_{i}(i=1,2)$. Further, a sub-sample of size $r\left(=n_{2} k^{-1}, k>1\right)$ is drawn from $n_{2}$ non-responding units using SRSWOR method of sampling and information on $r$ units is collected by personal interview. Hence, using Hansen and Hurwitz's [1] technique, the estimator for population mean $\overline{Y_{i}}$ based on $\left(n_{1}+r\right)$ units on study character $y_{i}$ is defined by

$\bar{y}_{i}^{\otimes}=\frac{n_{1}}{n} \bar{y}_{i(1)}+\frac{n_{2}}{n} \bar{y}_{i(2)}^{\prime}$,

where $\bar{y}_{i(1)}$ and $\bar{y}_{i(2)}^{\prime}$ are the means of study character $y_{i}$ based on $n_{1}$ and $r$ units respectively.

The estimator $\bar{y}_{i}^{\otimes}$ is unbiased and the variance of this estimator is given by

$V\left(\bar{y}_{i}^{\otimes}\right)=\frac{f}{n} S_{y i}^{2}+\frac{W_{2}(k-1)}{n} S_{y i}^{\otimes 2}$

where $f=(1-n / N), \mathrm{W}_{2}=N_{2} / N, S_{y i}^{2}$ and $S_{y i}^{\otimes 2}$ are the population mean squares of $y_{i}$ for the entire population and for the non-responding part of the population.

Let $\hat{R}\left(=\bar{y}_{1}^{\otimes} / \bar{y}_{2}^{\otimes}\right)$ denotes a conventional estimator for the estimation of ratio of two population means $R\left(=\overline{Y_{1}} / \overline{Y_{2}}\right)$.

In the case when population mean $(\bar{X})$ of auxiliary character is not known, a first phase sample of size $n^{\prime}(<N)$ is drawn from the population of size $N$ by using SRSWOR method of sampling and the population mean ( $\bar{X}$ ) is estimated by sample mean $\bar{x}^{\prime}$ based on $n^{\prime}$ units. Further, a second phase sample of size $n\left(<n^{\prime}\right)$ is drawn from first phase sample of size $n^{\prime}$ by using SRSWOR method of sampling and it is found that $n_{1}$ units respond and $n_{2}$ units do not respond in the sample of size $n$ for the study characters $y_{i}$. Again, a sub-sample of size $r\left(=n_{2} k^{-1}, k>1\right)$ is drawn from $n_{2}$ non-responding units by using SRSWOR method of sampling and the information on $r$ units is collected by personal interview. Hence, the mean of study character $y_{i}$ based on $\left(n_{1}+r\right)$ units is defined by equation (1). In this situation, conventional $\left(R_{1}\right)$ and alternative $\left(R_{2}\right)$ two phase sampling estimators for the estimation of ratio of two population means in the presence of non-response are defined as

$R_{1}=\hat{R} \frac{\bar{x}^{\otimes}}{\bar{x}^{\prime}} \quad$ and $\quad R_{2}=\hat{R} \frac{\bar{x}}{\bar{x}^{\prime}}$

where $\bar{x}=\frac{1}{n} \sum_{j=1}^{n} x_{j}, \bar{x}^{\otimes}=\frac{n_{1}}{n} \bar{x}_{1}+\frac{n_{2}}{n} \bar{x}_{2}^{\prime} ; \bar{x}_{1}$ and $\bar{x}_{2}^{\prime}$ are means of auxiliary characters $x$ based on $n_{1}$ and $r$ units corresponding to $n_{1}$ and $r$ units on study character $y_{i}$.

In such situation, improved classes of estimators for the estimation of ratio of two population means have been proposed by Khare and Sinha [4], which are given as follows:

$R_{3}=g_{1}(\hat{R}, u) \quad$ and $\quad R_{4}=g_{2}\left(\hat{R}, u^{\prime}\right)$

where $u=\frac{\bar{x}^{\otimes}}{\bar{x}^{\prime}}, u^{\prime}=\frac{\bar{x}}{\bar{x}^{\prime}}$.

If $\bar{X}$ is not known, but $\bar{Z}$, the population mean of the additional auxiliary character $z$ (closely related to $x$ ) is known which may be cheaper and less correlated to the study character in comparison to main auxiliary character i.e. $\rho_{y x}>\rho_{y z}$, then the population mean $\bar{X}$ is estimated by $\hat{\bar{X}}_{r}=\frac{\bar{x}^{\prime}}{\bar{z}^{\prime}} \bar{Z}$, where $\bar{x}^{\prime}$ and $\bar{z}^{\prime}$ are the first phase sample means based on $n^{\prime}(<N)$ units drawn from the population of size $N$ by using SRSWOR method of sampling. In this situation, the conventional and the alternative chain type estimators $\left(R_{5}, R_{6}\right)$ have been proposed by Khare et al. [11], which are given as follows: 
$R_{5}=\hat{R} \frac{\bar{x}^{\otimes}}{\bar{x}^{\prime}} \frac{\overline{\mathrm{z}}^{\prime}}{\overline{\mathrm{Z}}} \quad$ and $\quad R_{6}=\hat{R} \frac{\bar{x}}{\bar{x}^{\prime}} \frac{\overline{\mathrm{z}}^{\prime}}{\overline{\mathrm{Z}}}$

In such situation, we propose improved classes of chain type estimators $R_{c 1}$ and $R_{c 2}$ for the estimation of ratio of two population means using auxiliary characters in the presence of non-response, which are given as follows:

$R_{c 1}=f\left(\hat{R}, u_{1}, v\right) \quad$ and $\quad R_{c 2}=f\left(\hat{R}, u_{2}, v\right)$,

where $\hat{R}=\frac{\bar{y}_{1}^{\otimes}}{\bar{y}_{2}^{\otimes}}, u_{1}=\frac{\bar{x}^{*}}{\bar{x}^{\prime}}, \quad v=\frac{\bar{z}^{\prime}}{\bar{Z}}, \quad u_{2}=\frac{\bar{x}}{\bar{x}^{\prime}}$.

The functions $f\left(\hat{R}, u_{1}, v\right)$ and $f\left(\hat{R}, u_{2}, v\right)$ also satisfy the following regularity conditions:

(i) For any sampling design, whatever be the sample chosen, $\left(\hat{R}, u_{1}, v\right)$ and $\left(\hat{R}, u_{2}, v\right)$ assume values in a bounded, closed convex subset $G_{1}$ and $G_{2}$ respectively of three dimensional real space containing the points $(R, 1,1)$.

(ii) The functions $f\left(\hat{R}, u_{1}, v\right)$ and $f\left(\hat{R}, u_{2}, v\right)$ and their first and second partial derivatives exit and are continuous and bounded in $G_{1}$ and $G_{2}$ respectively.

\section{Bias and mean square error of the estimators $R_{c 1}$ and $R_{c 2}$}

Under the regularity conditions specified for the functions $f\left(\hat{R}, u_{1}, v\right)$ and $f\left(\hat{R}, u_{2}, v\right)$, bias and mean square error of the estimators $R_{c 1}$ and $R_{c 2}$ always exist. Now expanding $f\left(\hat{R}, u_{1}, v\right)$ and $f\left(\hat{R}, u_{2}, v\right)$ about the point $(R, 1,1)=A$ by using Taylor's series up to second order partial derivatives, we have

$$
\begin{aligned}
R_{c 1} & =f(A)+(\hat{R}-R) f_{1}(A)+\left(u_{1}-1\right) f_{2(1)}(A)+(v-1) f_{3}(A)+\frac{1}{2}\left\{(\hat{R}-R)^{2} f_{11(1)}\left(a_{1}^{\otimes}\right)\right. \\
& +\left(u_{1}-1\right)^{2} f_{22(1)}\left(a_{1}^{\otimes}\right)+(v-1)^{2} f_{33(1)}\left(a_{1}^{\otimes}\right)+2(\hat{R}-R)\left(u_{1}-1\right) f_{12(1)}\left(a_{1}^{\otimes}\right) \\
& \left.+2(\hat{R}-R)(v-1) f_{13(1)}\left(a_{1}^{\otimes}\right)+2\left(u_{1}-1\right)(v-1) f_{23(1)}\left(a_{1}^{\otimes}\right)\right\} .
\end{aligned}
$$

and

$$
\begin{aligned}
R_{c 2} & =f(A)+(\hat{R}-R) f_{1}(A)+\left(u_{2}-1\right) f_{2(2)}(A)+(v-1) f_{3}(A)+\frac{1}{2}\left\{(\hat{R}-R)^{2} f_{11(2)}\left(a_{2}^{\otimes}\right)\right. \\
& +\left(u_{2}-1\right)^{2} f_{22(2)}\left(a_{2}^{\otimes}\right)+(v-1)^{2} f_{33(2)}\left(a_{2}^{\otimes}\right)+2(\hat{R}-R)\left(u_{2}-1\right) f_{12(2)}\left(a_{2}^{\otimes}\right) \\
& \left.+2\left(u_{2}-1\right)(v-1) f_{23(2)}\left(a_{2}^{\otimes}\right)\right\} .
\end{aligned}
$$

After putting $f(A)=R, f_{1}(A)=1$ and $f_{11}(A)=0$, we observe

$$
\begin{aligned}
R_{c 1} & =\hat{R}+\left(u_{1}-1\right) f_{2(1)}(A)+(v-1) f_{3}(A)+\frac{1}{2}\left\{\left(u_{1}-1\right)^{2} f_{22(1)}\left(a_{1}^{\otimes}\right)+(v-1)^{2} f_{33(1)}\left(a_{1}^{\otimes}\right)\right. \\
& +2(\hat{R}-R)\left(u_{1}-1\right) f_{12(1)}\left(a_{1}^{\otimes}\right)+2(\hat{R}-R)(v-1) f_{13(1)}\left(a_{1}^{\otimes}\right) \\
& \left.+2\left(u_{1}-1\right)(v-1) f_{23(1)}\left(a_{1}^{\otimes}\right)\right\}, \\
R_{c 2} & =\hat{R}+\left(u_{2}-1\right) f_{2(2)}(A)+(v-1) f_{3}(A)+\frac{1}{2}\left\{\left(u_{2}-1\right)^{2} f_{22(2)}\left(a_{2}^{\otimes}\right)+(v-1)^{2} f_{33(2)}\left(a_{2}^{\otimes}\right)\right. \\
& +2(\hat{R}-R)\left(u_{2}-1\right) f_{12(2)}\left(a_{2}^{\otimes}\right)+2(\hat{R}-R)(v-1) f_{13(2)}\left(a_{2}^{\otimes}\right) \\
& \left.+2\left(u_{2}-1\right)(v-1) f_{23(2)}\left(a_{2}^{\otimes}\right)\right\},
\end{aligned}
$$

where 


$$
\begin{array}{lrl}
f_{1}(A)=\left(\frac{\partial f\left(a_{i}\right)}{\partial \hat{R}}\right)_{A}, & f_{2(i)}(A)=\left(\frac{\partial f\left(a_{i}\right)}{\partial u_{i}}\right)_{A}, & f_{3}(A)=\left(\frac{\partial f\left(a_{i}\right)}{\partial v}\right)_{A}, \\
f_{11(i)}\left(a_{i}^{\otimes}\right)=\frac{\partial^{2} f\left(a_{i}^{\otimes}\right)}{\partial \hat{R}^{2}}, & f_{22(i)}\left(a_{i}^{\otimes}\right)=\frac{\partial^{2} f\left(a_{i}^{\otimes}\right)}{\partial u_{i}^{2}}, & f_{33(i)}\left(a_{i}^{\otimes}\right)=\frac{\partial^{2} f\left(a_{i}^{\otimes}\right)}{\partial v^{2}}, \\
f_{12(i)}\left(a_{i}^{\otimes}\right)=\frac{\partial^{2} f\left(a_{i}^{\otimes}\right)}{\partial \hat{R} \partial u_{i}}, & f_{13(i)}\left(a_{i}^{\otimes}\right)=\frac{\partial^{2} f\left(a_{i}^{\otimes}\right)}{\partial \hat{R} \partial v}, & f_{23(i)}\left(a_{i}^{\otimes}\right)=\frac{\partial^{2} f\left(a_{i}^{\otimes}\right)}{\partial u_{i} \partial v},
\end{array}
$$

$a_{i}=\left(w, u_{i}, v\right), a_{i}^{\otimes}=\left(w^{\otimes}, u_{i}^{\otimes}, v^{\otimes}\right), w^{\otimes}=R+\theta(\hat{R}-R), u_{i}^{\otimes}=1+\theta_{i}\left(u_{i}-1\right), \quad v=1+\theta_{0}(v-1)$,

such that $0<\theta, \theta_{i}, \theta_{0}<1, \quad \forall \quad i=1,2$

Let $\bar{y}_{1}^{\otimes}=\overline{Y_{1}}\left(1+\varepsilon_{0}\right), \bar{y}_{2}^{\otimes}=\overline{Y_{2}}\left(1+\varepsilon_{1}\right), \bar{x}^{\otimes}=\bar{X}\left(1+\varepsilon_{2}\right), \bar{x}=\bar{X}\left(1+\varepsilon_{3}\right), \bar{x}^{\prime}=\bar{X}\left(1+\varepsilon_{4}\right), \bar{z}^{\prime}=\bar{Z}\left(1+\varepsilon_{5}\right)$,

such that $\quad E\left(\varepsilon_{h}\right)=0$ and $\quad\left|\varepsilon_{h}\right|<1 \quad \forall \quad h=0,1,2,3,4,5$.

Now using SRSWOR method of sampling, we have

$$
\begin{aligned}
& E\left(\varepsilon_{0}^{2}\right)=\frac{f}{n} C_{y 1}^{2}+\frac{W_{2}(k-1)}{n} C_{y 1}^{\otimes 2}, E\left(\varepsilon_{1}^{2}\right)=\frac{f}{n} C_{y 2}^{2}+\frac{W_{2}(k-1)}{n} C_{y 2}^{\otimes 2} \quad E\left(\varepsilon_{2}^{2}\right)=\frac{f}{n} C_{x}^{2}+\frac{W_{2}(k-1)}{n} C_{x}^{\otimes 2}, \quad E\left(\varepsilon_{3}^{2}\right)=\frac{f}{n} C_{x}^{2}, \\
& E\left(\varepsilon_{4}^{2}\right)=\frac{f^{\prime}}{n^{\prime}} C_{x}^{2}, \quad E\left(\varepsilon_{5}^{2}\right)=\frac{f^{\prime}}{n^{\prime}} C_{z}^{2}, \quad E\left(\varepsilon_{0} \varepsilon_{1}\right)=\frac{f}{n} C_{y 1 y 2}+\frac{W_{2}(k-1)}{n} C_{y 1 y 2}^{\otimes}, E\left(\varepsilon_{0} \varepsilon_{2}\right)=\frac{f}{n} C_{y 1 x}+\frac{W_{2}(k-1)}{n} C_{y 1 x}^{\otimes}, \\
& E\left(\varepsilon_{0} \varepsilon_{3}\right)=\frac{f}{n} C_{y 1 x}, E\left(\varepsilon_{0} \varepsilon_{4}\right)=\frac{f^{\prime}}{n^{\prime}} C_{y 1 x}, E\left(\varepsilon_{0} \varepsilon_{5}\right)=\frac{f^{\prime}}{n^{\prime}} C_{y 1 z}, \quad E\left(\varepsilon_{1} \varepsilon_{2}\right)=\frac{f}{n} C_{y 2 x}+\frac{W_{2}(k-1)}{n} C_{y 2 x}^{\otimes}, E\left(\varepsilon_{1} \varepsilon_{3}\right)=\frac{f}{n} C_{y 2 x}, \\
& E\left(\varepsilon_{1} \varepsilon_{4}\right)=\frac{f^{\prime}}{n^{\prime}} C_{y 2 x}, \quad E\left(\varepsilon_{1} \varepsilon_{5}\right)=\frac{f^{\prime}}{n^{\prime}} C_{y 2 z} \quad E\left(\varepsilon_{2} \varepsilon_{4}\right)=\frac{f^{\prime}}{n^{\prime}} C_{x}^{2}, \quad E\left(\varepsilon_{2} \varepsilon_{5}\right)=\frac{f^{\prime}}{n^{\prime}} C_{x z}, \\
& E\left(\varepsilon_{3} \varepsilon_{5}\right)=\frac{f^{\prime}}{n^{\prime}} C_{x z}, \quad E\left(\varepsilon_{4} \varepsilon_{5}\right)=\frac{f^{\prime}}{n^{\prime}} C_{x z} .
\end{aligned}
$$

The expressions for bias and mean square error of the proposed estimators $R_{c 1}$ and $R_{c 2}$ upto term of order $\left(n^{-1}\right)$ are given as

$$
\begin{aligned}
\operatorname{Bias}\left(R_{c 1}\right) & =\frac{1}{2}\left[\left(\frac{1}{n}-\frac{1}{n^{\prime}}\right)\left\{f_{22(1)}\left(a_{1}^{\otimes}\right) C_{x}^{2}+2 R f_{12(1)}\left(a_{1}^{\otimes}\right) \Delta\right\}\right. \\
& +\frac{W_{2}(k-1)}{n}\left\{f_{22(1)}\left(a_{1}^{\otimes}\right) C_{x}^{\otimes 2}+2 R f_{12(1)}\left(a_{1}^{\otimes}\right) \Delta^{\otimes}\right\} \\
& \left.+\frac{f^{\prime}}{n^{\prime}}\left\{f_{33(1)}\left(a_{1}^{\otimes}\right) C_{z}^{2}+2 R f_{13(1)}\left(a_{1}^{\otimes}\right) \Delta_{1}\right\}\right],
\end{aligned}
$$

$$
\begin{aligned}
\operatorname{Bias}\left(R_{c 2}\right) & =\frac{1}{2}\left[\left(\frac{1}{n}-\frac{1}{n^{\prime}}\right)\left\{f_{22(2)}\left(a_{2}^{\otimes}\right) C_{x}^{2}+2 R f_{12(2)}\left(a_{2}^{\otimes}\right) \Delta\right\}\right. \\
& \left.+\frac{f^{\prime}}{n^{\prime}}\left\{f_{33(2)}\left(a_{2}^{\otimes}\right) C_{z}^{2}+2 R f_{13(2)}\left(a_{2}^{\otimes}\right) \Delta_{1}\right\}\right]
\end{aligned}
$$

and

$$
\begin{aligned}
\operatorname{MSE}\left(R_{c 1}\right) & =\operatorname{MSE}(\hat{R})+\left[f_{2(1)}^{2}(A)\left\{\left(\frac{1}{n}-\frac{1}{n^{\prime}}\right) C_{x}^{2}+\frac{W_{2}(k-1)}{n} C_{x}^{\otimes 2}\right\}+\right. \\
& \left.+2 f_{2(1)}(A) R\left\{\left(\frac{1}{n}-\frac{1}{n^{\prime}}\right) \Delta+\frac{W_{2}(k-1)}{n} \Delta^{\otimes}\right\}+\frac{f^{\prime}}{n^{\prime}} f_{3}(A)\left(f_{3}(A) C_{z}^{2}+2 R \Delta_{1}\right)\right],
\end{aligned}
$$




$$
\begin{aligned}
\operatorname{MSE}\left(R_{c 2}\right) & =\operatorname{MSE}(\hat{R})+\left[\left(\frac{1}{n}-\frac{1}{n^{\prime}}\right) f_{2(2)}(A)\left(f_{2(2)}(A) C_{x}^{2}+2 R \Delta\right)\right. \\
& \left.+\frac{f^{\prime}}{n^{\prime}} f_{3}(A)\left(f_{3}(A) C_{z}^{2}+2 R \Delta_{1}\right)\right]
\end{aligned}
$$

where

$$
\operatorname{MSE}(\hat{R})=R^{2}\left[\frac{f}{n}\left(C_{y 1}^{2}+C_{y 2}^{2}-2 \rho C_{y 1} C_{y 2}\right)+\frac{W_{2}(k-1)}{n}\left(C_{y 1}^{\otimes 2}+C_{y 2}^{\otimes 2}-2 \rho^{\otimes} C_{y 1}^{\otimes} C_{y 2}^{\otimes}\right)\right],
$$

$\Delta=\left(\rho_{y 1 x} C_{y 1} C_{x}-\rho_{y 2 x} C_{y 2} C_{x}\right), \quad \quad \Delta^{\otimes}=\left(\rho_{y 1 x}^{\otimes} C_{y 1}^{\otimes} C_{x}^{\otimes}-\rho_{y 2 x}^{\otimes} C_{y 2}^{\otimes} C_{x}^{\otimes}\right)$,

$\Delta_{1}=\left(\rho_{y 1 z} C_{y 1} C_{z}-\rho_{y 2 z} C_{y 2} C_{z}\right), C_{y i}=\frac{S_{y i}}{\bar{Y}_{i}}, C_{y i}^{\otimes}=\frac{S_{y i}^{\otimes}}{\overline{Y_{i}}}, C_{x}=\frac{S_{x}}{\bar{X}}, C_{x}^{\otimes}=\frac{S_{x}^{\otimes}}{\bar{X}}, C_{z}=\frac{S_{z}}{\bar{Z}}, \quad f^{\prime}=\left(1-\frac{n^{\prime}}{N}\right)$,

$S_{y i}^{2}=\frac{1}{N-1} \sum_{j=1}^{N}\left(Y_{i j}-\overline{Y_{i}}\right)^{2}, \quad S_{y i}^{\otimes 2}=\frac{1}{N_{2}-1} \sum_{j=1}^{N_{2}}\left\{Y_{i j}-\bar{Y}_{i(2)}\right\}^{2}, \quad S_{x}^{2}=\frac{1}{N-1} \sum_{j=1}^{N}\left(X_{j}-\bar{X}\right)^{2}$

$S_{x}^{\otimes 2}=\frac{1}{N_{2}-1} \sum_{j=1}^{N_{2}}\left\{X_{j}-\bar{X}_{(2)}\right\}^{2}$ and $S_{z}^{2}=\frac{1}{N-1} \sum_{j=1}^{N}\left(Z_{j}-\bar{Z}\right)^{2}, \rho, \rho_{y i x}$ and $\rho_{y i z}$ denote the correlation coefficients between $\left(y_{1}, y_{2}\right),\left(y_{i}, x\right)$ and $\left(y_{i}, z\right)$, for the entire population, $\rho^{\otimes}$ and $\rho_{y i x}^{\otimes}$ denote the correlation coefficients between $\left(y_{1}, y_{2}\right)$ and $\left(y_{i}, x\right)$ for the non-responding part of the population and $\left(\bar{Y}_{i(2)}, \bar{X}_{(2)}\right)$ denote the means of the study character $y$ and auxiliary character $x$ for the non-responding part of the population

The optimum values of $f_{2(1)}(A), f_{3}(A)$ and $f_{2(2)}(A)$, which minimize the $\operatorname{MSE}\left(R_{c 1}\right)$ and $M S E\left(R_{c 2}\right)$ are given as follows:

$$
\begin{aligned}
& f_{2(1) o p t}(A)=-\frac{R\left\{\left(\frac{1}{n}-\frac{1}{n^{\prime}}\right) \Delta+\frac{W_{2}(k-1)}{n} \Delta^{\otimes}\right\}}{\left\{\left(\frac{1}{n}-\frac{1}{n^{\prime}}\right) C_{x}^{2}+\frac{W_{2}(k-1)}{n} C_{x}^{\otimes 2}\right\}}, \\
& f_{3 \text { opt }}(A)=-\frac{R\left(\rho_{y 1 z} C_{y 1}-\rho_{y 2 z} C_{y 2}\right)}{C_{z}}
\end{aligned}
$$

and

$f_{2(2) o p t}(A)=-\frac{R\left(\rho_{y 1 x} C_{y 1}-\rho_{y 2 x} C_{y 2}\right)}{C_{x}}$

By putting the optimum values of $f_{2(1)}(A), f_{3}(A)$ and $f_{2(2)}(A)$ in the expression of (13) and (14), the minimum values of $\operatorname{MSE}\left(R_{c 1}\right)$ and $\operatorname{MSE}\left(R_{c 2}\right)$ are given as follows:

$$
\operatorname{MSE}\left(R_{c 1}\right)_{\min }=\operatorname{MSE}(\hat{R})-R^{2}\left[\frac{\left\{\left(\frac{1}{n}-\frac{1}{n^{\prime}}\right) \Delta+\frac{W_{2}(k-1)}{n} \Delta^{\otimes}\right\}^{2}}{\left\{\left(\frac{1}{n}-\frac{1}{n^{\prime}}\right) C_{x}^{2}+\frac{W_{2}(k-1)}{n} C_{x}^{\otimes 2}\right\}}+\frac{f^{\prime}}{n^{\prime}}\left(\frac{\Delta_{1}}{C_{z}}\right)^{2}\right] .
$$


$\operatorname{MSE}\left(R_{c 2}\right)_{\min }=\operatorname{MSE}(\hat{R})-R^{2}\left[\left(\frac{1}{n}-\frac{1}{n^{\prime}}\right)\left(\frac{\Delta}{C_{x}}\right)^{2}+\frac{f^{\prime}}{n^{\prime}}\left(\frac{\Delta_{1}}{C_{z}}\right)^{2}\right]$.

If $\frac{\Delta}{C_{x}^{2}}\left\{=\left(\beta_{y 1 x} / R_{1}-\beta_{y 2 x} / R_{2}\right)\right\}=\frac{\Delta^{\otimes}}{C_{x}^{\otimes 2}}\left\{=\left(\beta_{y 1 x}^{\otimes} / R_{1}-\beta_{y 2 x}^{\otimes} / R_{2}\right)\right\}$ for $\beta_{y 1 x}=\beta_{y 1 x}^{\otimes}$ and $\beta_{y 2 x}=\beta_{y 2 x}^{\otimes}$

then $\frac{\left\{\left(\frac{1}{n}-\frac{1}{n^{\prime}}\right) \Delta+\frac{W_{2}(k-1)}{n} \Delta^{\otimes}\right\}}{\left\{\left(\frac{1}{n}-\frac{1}{n^{\prime}}\right) C_{x}^{2}+\frac{W_{2}(k-1)}{n} C_{x}^{\otimes 2}\right\}}=\frac{\Delta}{C_{x}^{2}}$ and in this case, we have

$\operatorname{MSE}\left(R_{c 1}\right)_{\min }=\operatorname{MSE}(\hat{R})-R^{2}\left[\left(\frac{1}{n}-\frac{1}{n^{\prime}}\right)\left(\frac{\Delta}{C_{x}}\right)^{2}+\frac{W_{2}(k-1)}{n}\left(\frac{\Delta^{\otimes}}{C_{x}^{\otimes}}\right)^{2}+\frac{f^{\prime}}{n^{\prime}}\left(\frac{\Delta_{1}}{C_{z}}\right)^{2}\right]$.

where $\beta_{y 1 x}=\frac{S_{y 1 x}}{S_{x}^{2}}, \beta_{y 2 x}=\frac{S_{y 2 x}}{S_{x}^{2}}, \beta_{y 1 x}^{\otimes}=\frac{S_{y 1 x}^{\otimes}}{S_{x}^{\otimes 2}}, \beta_{y 2 x}^{\otimes}=\frac{S_{y 2 x}^{\otimes}}{S_{x}^{\otimes 2}}, R_{1}=\frac{\overline{Y_{1}}}{\bar{X}}$ and $R_{2}=\frac{\overline{Y_{2}}}{\bar{X}}$.

So, the minimum mean square error of $R_{c 1}$ which is given in equation (13) may also be approximated to minimum mean square error of $R_{c 1}$ which is given in equation (21).

The optimum values of $f_{2(1)}(A), f_{3}(A)$ and $f_{2(2)}(A)$ are in the form of the value of unknown constants. Sometimes these unknown constants are in the form of some unknown parameters. In these situation, the optimum values of the constants may be obtained from past data of the parameter [Reddy [12]], or by estimating the parameters included in the optimum value of constant on the basis of sample values. The minimum values of the mean square error of the estimators upto the terms of order $n^{-1}$ are unchanged if we estimate the optimum values of the constants by using the sample values [Srivastava and Jhajj [13]].

The parametric functions $f\left(\hat{R}, u_{1}, v\right)$ and $f\left(\hat{R}, u_{2}, v\right)$ satisfy the regularity conditions (i) and (ii) can generate a class of asymptotic estimators. The class of such type estimator is very large. Some members of the proposed class of chain type estimators are given as follows:

$R_{b 1}=\hat{R} u_{1}^{\alpha_{1}} v^{\alpha_{2}}, \quad R_{b 2}=\hat{R} u_{2}^{\beta_{1}} v^{\beta_{2}}$,

$R_{b 3}=\hat{R}\left[a u_{1}^{\alpha_{1}}+(1-a) v^{\alpha_{2}}\right], \quad R_{b 4}=\hat{R}\left[a u_{2}^{\beta_{1}}+(1-a) v^{\beta_{2}}\right]$,

$R_{b 5}=\hat{R}\left[1+\alpha_{1}\left(u_{1}-1\right)+\alpha_{2}(v-1)\right]$ and $R_{b 6}=\hat{R}\left[1+\beta_{1}\left(u_{2}-1\right)+\beta_{2}(v-1)\right]$,

where $\alpha_{1}, \alpha_{2}, \beta_{1} \beta_{1}$ and $a$ are constants.

The $M S E(.)_{\min }$ of all the members belonging to the improved classes of chain type estimators $R_{c 1}$ and $R_{c 2}$ will be same as given in (13) and (14).

The expressions for the mean square error of the estimators $R_{1}, R_{2}, R_{3}, R_{4} R_{5}$ and $R_{6}$ are given as follows:

$$
\begin{aligned}
& \operatorname{MSE}\left(R_{1}\right)=\operatorname{MSE}(\hat{R})+R^{2}\left[\left(\frac{1}{n}-\frac{1}{n^{\prime}}\right)\left\{C_{x}^{2}+2 \Delta\right\}+\frac{W_{2}(k-1)}{n}\left\{C_{x}^{\otimes 2}+2 \Delta^{\otimes}\right\}\right], \\
& \operatorname{MSE}\left(R_{2}\right)=\operatorname{MSE}(\hat{R})+R^{2}\left[\left(\frac{1}{n}-\frac{1}{n^{\prime}}\right)\left\{C_{x}^{2}+2 \Delta\right\}\right], \\
& \operatorname{MSE}\left(R_{3}\right)_{\min }=\operatorname{MSE}(\hat{R})-R^{2}\left[\left(\frac{1}{n}-\frac{1}{n^{\prime}}\right)\left(\frac{\Delta}{C_{x}}\right)^{2}+\frac{W_{2}(k-1)}{n}\left(\frac{\Delta^{\otimes}}{C_{x}^{\otimes}}\right)^{2}\right], \\
& \operatorname{MSE}\left(R_{4}\right)_{\min }=\operatorname{MSE}(\hat{R})-R^{2}\left[\left(\frac{1}{n}-\frac{1}{n^{\prime}}\right)\left(\frac{\Delta}{C_{x}}\right)^{2}\right], \\
& \operatorname{MSE}\left(R_{5}\right)=\operatorname{MSE}(\hat{R})+R^{2}\left[\left(\frac{1}{n}-\frac{1}{n^{\prime}}\right)\left(C_{x}^{2}+2 \Delta\right)+\frac{W_{2}(k-1)}{n}\left(C_{x}^{\otimes 2}+2 \Delta^{\otimes}\right)\right.
\end{aligned}
$$




$$
\left.+\frac{f^{\prime}}{n^{\prime}}\left(C_{z}^{2}+2 \Delta_{1}\right)\right]
$$

and

$$
\operatorname{MSE}\left(R_{6}\right)=\operatorname{MSE}(\hat{R})+R^{2}\left[\left(\frac{1}{n}-\frac{1}{n^{\prime}}\right)\left(C_{x}^{2}+2 \Delta\right)+\frac{f^{\prime}}{n^{\prime}}\left(C_{z}^{2}+2 \Delta_{1}\right)\right] .
$$

\section{Determination of $n^{\prime}, n$ and $k$ for the fixed $\operatorname{cost} C \leq C_{0}$}

Let $C_{0}$ denotes the total cost (fixed) of the survey apart from overhead cost. The cost function $C^{\prime}$ can be written as

$C^{\prime}=\left(e_{1}^{\prime}+e_{2}^{\prime}\right) n^{\prime}+e_{1} n+e_{2} n_{1}+e_{3} \frac{n_{2}}{k}$

where

$e_{1}^{\prime}-$ the cost per unit of identifying and observing auxiliary character $x$ at the first phase,

$e_{2}^{\prime}-$ the cost per unit of obtaining information on additional auxiliary character $z$ at the first phase,

$e_{1}-$ the cost per unit of mailing questionnaire visiting the units at the second phase,

$e_{2}-$ the cost per unit of collecting and processing data for the study characters $y_{1}$ and $y_{2}$ obtained from $n_{1}$ responding units and

$e_{3}$ - the cost per unit of obtaining and processing data for the study characters $y_{1}$ and $y_{2}$ (after extra effort) from the sub-sampled units.

It is to be noted that $e_{2}^{\prime}<e_{1}^{\prime}<e_{1}<e_{2}<e_{3}$.

The expected total cost of the survey apart from overhead cost is given as

$C=\left(e_{1}^{\prime}+e_{2}^{\prime}\right) n^{\prime}+n\left(e_{1}+e_{2} W_{1}+e_{3} \frac{W_{2}}{k}\right)$,

where $W_{1}=\frac{N_{1}}{N}$ is the response rate in the population.

The $\operatorname{MSE}(R(l)): l=1,2,3,4$ can be expressed in terms of the notations $R^{2} S_{0 l}, R^{2} S_{1 l}, R^{2} S_{2 l}$ and $R^{2} S_{3 l}$, which is given as follows:

$\operatorname{MSE}(R(l))=\left[\frac{1}{n} R^{2} S_{0 l}+\frac{1}{n^{\prime}} R^{2} S_{1 l}+\frac{k}{n} R^{2} S_{2 l}-\frac{1}{N} R^{2} S_{3 l}\right]$,

where $R(1)=R_{5}, R(2)=R_{6}, R(3)=R_{c 1}, R(4)=R_{c 2}$ and $R^{2} S_{0 l}, R^{2} S_{1 l}, R^{2} S_{2 l}$ and $R^{2} S_{3 l}$ are respectively the coefficients of terms of $n^{-1}, n^{\prime-1}, k n^{-1}$ and $N^{-1}$ in the expressions of $\operatorname{MSE}(R(l))$. Here $\operatorname{MSE}\left(R_{c 1}\right)$ are taken on the assumption that $\beta_{y 1 x}=\beta_{y 1 x}^{\otimes}$ and $\beta_{y 2 x}=\beta_{y 2 x}^{\otimes}$ for simplicity.

Now, we define a function $\varphi$ for minimizing the $\operatorname{MSE}(R(l))$ and also to obtain the optimum values of $n^{\prime}, n$ and $k$ in the case of the fixed cost $C \leq C_{0}$, which is given as follows:

$\varphi=\operatorname{MSE}(R(l))+\lambda_{l}\left\{\left(e_{1}^{\prime}+e_{2}^{\prime}\right) n^{\prime}+n\left(e_{1}+e_{2} W_{1}+e_{3} \frac{W_{2}}{k}\right)-C_{0}\right\}$,

where $\lambda_{l}$ is Lagrange's multiplier.

After differentiating $\varphi$ with respect to $n^{\prime}, n$ and $k$ and equating to zero, we have

$$
n^{\prime}=R \sqrt{\frac{S_{1 l}}{\lambda_{l}\left(e_{1}^{\prime}+e_{2}^{\prime}\right)}},
$$




$$
n=R \sqrt{\frac{\left(S_{0 l}+k S_{2 l}\right)}{\lambda_{l}\left(e_{1}+e_{2} W_{1}+e_{3} \frac{W_{2}}{k}\right)}}
$$

and

$$
k_{o p t}=\sqrt{\frac{e_{3} W_{2} S_{o l}}{\left(e_{1}+e_{2} W_{1}\right) S_{2 l}}},
$$

where

$$
\sqrt{\lambda_{l}}=\frac{R}{C_{0}}\left[\sqrt{S_{1 l}\left(e_{1}^{\prime}+e_{2}^{\prime}\right)}+\sqrt{\left(S_{0 l}+k_{o p t} S_{2 l}\right)\left(e_{1}+e_{2} W_{1}+e_{3} \frac{W_{2}}{k_{o p t}}\right)}\right] .
$$

Here we observe that for the optimum values of $n^{\prime}, n$ and $k$, the determinant of the matrix of second order derivative of $\varphi$ with respect to $n^{\prime}, n$ and $k$ is positive, which shows that the optimum values of $n^{\prime}$ and $n$ given by (35) and (36) and using (37) and (38) under the condition $C \leq C_{0}$ minimize the mean square error of $R(l)$.

So for the optimum values of $n^{\prime}, n$ and $k$, the minimum mean square error of $R(l)$ is given by

$$
\operatorname{MSE}(R(l))_{\min }=R^{2}\left[\frac{1}{C_{0}}\left\{\sqrt{S_{1 l}\left(e_{1}^{\prime}+e_{2}^{\prime}\right)}+\sqrt{\left(S_{0 l}+k_{o p t} S_{2 l}\right)\left(e_{1}+e_{2} W_{1}+e_{3} \frac{W_{2}}{k_{o p t}}\right)}\right\}^{2}-\frac{1}{N} S_{3 l}\right]
$$

After neglecting the term of order $\left(N^{-1}\right)$, we have

$$
\operatorname{MSE}(R(l))_{\min }=R^{2}\left[\frac{1}{C_{0}}\left\{\sqrt{S_{1 l}\left(e_{1}^{\prime}+e_{2}^{\prime}\right)}+\sqrt{\left(S_{0 l}+k_{\text {opt }} S_{2 l}\right)\left(e_{1}+e_{2} W_{1}+e_{3} \frac{W_{2}}{k_{\text {opt }}}\right)}\right\}^{2}\right]
$$

\section{Determination of $n^{\prime}, n$ and $k$ for the specified variance $V=V_{0}$}

Let $V_{0}$ denotes the specified variance of the estimator $R(l)$, which is fixed in advance. So, we have

$V_{0}=\left[\frac{1}{n} R^{2} S_{0 l}+\frac{1}{n^{\prime}} R^{2} S_{1 l}+\frac{k}{n} R^{2} S_{2 l}-\frac{1}{N} R^{2} S_{3 l}\right]$.

For minimizing the average total cost $C$ for the specified variance (i.e., $\left.\operatorname{MSE}(R(l))=V_{0}\right)$ of the estimators $R(l)$ and for obtaining the optimum values of $n^{\prime}, n$ and $k$, we define a function $\psi$ which is written as follows:

$\psi=\left\{\left(e_{1}^{\prime}+e_{2}^{\prime}\right) n^{\prime}+n\left(e_{1}+e_{2} W_{1}+e_{3} \frac{W_{2}}{k}\right)\right\}+\mu_{l}\left(\operatorname{MSE}(R(l))-V_{0}\right)$,

where $\mu_{l}$ is Lagrange's multiplier.

Now after differentiating $\psi$ with respect to $n^{\prime}, n$ and $k$ and equating to zero, we get

$$
\begin{aligned}
& n^{\prime}=R \sqrt{\frac{\mu_{l} S_{1 l}}{\left(e_{1}^{\prime}+e_{2}^{\prime}\right)}}, \\
& n=R \sqrt{\frac{\mu_{l}\left(S_{0 l}+k S_{2 l}\right)}{\left(e_{1}+e_{2} W_{1}+e_{3} \frac{W_{2}}{k}\right)}}
\end{aligned}
$$

and 
$k_{\text {opt }}=\sqrt{\frac{S_{0 l} e_{3} W_{2}}{S_{2 l}\left(e_{1}+e_{2} W_{1}\right)}}$,

where $\sqrt{\mu_{l}}$ is given by

$\sqrt{\mu_{l}}=R \frac{\left[\sqrt{\left(e_{1}^{\prime}+e_{2}^{\prime}\right) S_{1 l}}+\sqrt{\left(S_{0 l}+k_{o p t} S_{2 l}\right)\left(e_{1}+e_{2} W_{1}+e_{3} \frac{W_{2}}{k_{o p t}}\right)}\right]}{\left[V_{0}+\frac{1}{N} R^{2} S_{3 l}\right]}$.

After putting the value of $k_{\text {opt }}$ and the optimum value of $\sqrt{\mu_{l}}$ from (45) and (46) in (43) and (44) respectively, we find the optimum values of $n^{\prime}$ and $n$. Further, we observe that for the optimum values of $n^{\prime}, n$ and $k$, the determinant of the matrix of the second order derivative of $\psi$ with respect to $n^{\prime}, n$ and $k$ is positive. The minimum expected total cost to be incurred on the use of $R(l)$ for the specified variance, will be given by

$C(R(l))_{\min }=\frac{R^{2}\left[\sqrt{\left(e_{1}^{\prime}+e_{2}^{\prime}\right) S_{1 l}}+\sqrt{\left(S_{0 l}+k_{o p t} S_{2 l}\right)\left(e_{1}+e_{2} W_{1}+e_{3} \frac{W_{2}}{k_{o p t}}\right)}\right]^{2}}{\left[V_{0}+\frac{1}{N} R^{2} S_{3 l}\right]}$

After neglecting the term of order $\left(N^{-1}\right)$, we have

$C(R(l))_{\min }=\frac{R^{2}\left[\sqrt{\left(e_{1}^{\prime}+e_{2}^{\prime}\right) S_{1 l}}+\sqrt{\left(S_{0 l}+k_{\text {opt }} S_{2 l}\right)\left(e_{1}+e_{2} W_{1}+e_{3} \frac{W_{2}}{k_{\text {opt }}}\right)}\right]^{2}}{V_{0}}$.

\section{An empirical study}

The present data have been taken from the paper of Khare and Sinha [5]. This data belong to the data on physical growth of upper socio- economic group of 95 school going children of Varanasi under an ICMR study, Department of Pediatrics, BHU during 1983-84 has been taken under study. The first 25\% (i.e. 24 children) units have been considered as non-response units. The values of parameters related to the study characters $y_{1}$ (the height of children in $\mathrm{cm}$.) and $y_{2}$ (weight of children in $\mathrm{kg}$ ), the auxiliary character $x$ (chest circumference of the children in $\mathrm{cm}$ ) and additional auxiliary character $z$ (skull circumference of children in $\mathrm{cm}$ ) have been given as follows:
$\overline{Y_{1}}=115.9526$,
$\overline{Y_{2}}=19.4968$,
$\bar{X}=55.8611$,
$\bar{Z}=51.1726$,
$C_{y 1}=0.05146$,
$C_{y 2}=0.15613$,
$C_{x}=0.05860$,
$C_{z}=0.03006$,
$C_{y 1}^{\otimes}=0.04402$,
$C_{y 2}^{\otimes}=0.12075$,
$C_{x}^{\otimes}=0.05402$,
$\rho_{y 1 x}=0.620$,
$\rho_{y 2 x}=0.846$,
$\rho_{y 1 z}=0.374$,
$\rho_{y 2 z}=0.328$,
$\rho_{y 1 x}^{\otimes}=0.401$,
$\rho_{y 2 x}^{\otimes}=0.729$,
$\rho=0.713$
$\rho^{\otimes}=0.678$.

The problem considered is to estimate the ratio between height and weight of the male children aged 6-7 years using chest circumference as the auxiliary character and skull circumference as the additional auxiliary character. 
Table 1: Relative efficiency (in \%) of the estimators with respect to $\hat{R}$ for different values of $k$

\begin{tabular}{lccc}
\hline & \multicolumn{1}{c}{$N=95}$, & $n^{\prime}=75$, & $n=55$ \\
\cline { 2 - 4 } Estimators & $1 / 4$ & $1 / \mathrm{k}$ & $1 / 2$ \\
\cline { 2 - 4 } & $100.00(0.00875)$ & $100.00(0.00724)$ & $100.00(0.00573)$ \\
$n_{R}$ & $173.49(0.00505)$ & $168.63(0.00429)$ & $161.70(0.00354)$ \\
$R_{1}$ & $119.44(0.00733)$ & $124.49(0.00582)$ & $133.10(0.00430)$ \\
$R_{2}$ & $186.75(0.00469)$ & $182.37(0.00397)$ & $176.48(0.00325)$ \\
$R_{3}$ & $124.47(0.00703)$ & $131.64(0.00552)$ & $142.95(0.00401)$ \\
$R_{4}$ & $177.03(0.00494)$ & $172.69(0.00419)$ & $166.45(0.00344)$ \\
$R_{5}$ & $121.12(0.00723)$ & $126.69(0.00571)$ & $136.31(0.00420)$ \\
$R_{6}$ & $190.89(0.00459)$ & $187.16(0.00387)$ & $182.18(0.00314)$ \\
$R_{c 1}$ & $126.29(0.00693)$ & $133.64(0.00542)$ & $146.67(0.00391)$ \\
$R_{c 2}$ & &
\end{tabular}

Figures in parenthesis give the MSE (.).

From table 1, it has been observed that for the fixed values of $\left(n^{\prime}, n\right)$ and different values of $k$, the proposed classes of chain type estimators $\left(R_{c 1}, R_{c 2}\right)$ are more efficient than the corresponding estimators $\left(R_{1}, R_{2}\right),\left(R_{3}, R_{4}\right)$ and $\left(R_{5}, R_{6}\right)$. However, $R_{c 1}$ is more efficient than $R_{c 2}$. The values of mean square error of all the estimators decrease as the values of $k$ decrease.

Table 2: Relative efficiency (in \%) of the estimators with respect to $\hat{R}$ for the fixed cost $C_{0}=$ Rs. 300

\begin{tabular}{|c|c|c|c|c|c|}
\hline & $e_{1}^{\prime}=R s .1 .50$, & $e_{2}^{\prime}=R s . \quad 0.10$ & $e_{1}=R s .5$ & $e_{2}=R s .9$, & $e_{3}=R s .38$ \\
\hline Estimators & $k_{\text {opt }}$ & $\begin{array}{c}n_{o p t}^{\prime} \\
\text { (approx.) }\end{array}$ & $\begin{array}{c}n_{o p t} \\
\text { (approx.) }\end{array}$ & & $\begin{array}{l}\text { Relative efficiency } \\
\text { (in \%) }\end{array}$ \\
\hline$\hat{R}$ & 2.14 & --- & 19 & & $100.00(1.2338)$ \\
\hline$R_{1}$ & 2.07 & 46 & 14 & & $125.87(0.9802)$ \\
\hline$R_{2}$ & 1.31 & 44 & 12 & & $117.49(1.0501)$ \\
\hline$R_{3}$ & 1.82 & 54 & 13 & & $143.89(0.8574)$ \\
\hline$R_{4}$ & 1.05 & 53 & 11 & & $136.22(0.9057)$ \\
\hline$R_{5}$ & 2.07 & 42 & 14 & & $127.78(0.9655)$ \\
\hline$R_{6}$ & 1.31 & 41 & 12 & & $119.22(1.0349)$ \\
\hline$R_{c 1}$ & 1.82 & 50 & 13 & & $145.56(0.8476)$ \\
\hline$R_{c 2}$ & 1.05 & 49 & 11 & & $137.75(0.8957)$ \\
\hline
\end{tabular}

Figures in parenthesis give the MSE (.), Rs. -Rupees (Indian Currency)

From table 2, it has been observed that for the fixed cost, the proposed classes of chain type estimators $\left(R_{c 1}, R_{c 2}\right)$ have also less mean square error in comparison to the corresponding estimators $\left(R_{1}, R_{2}\right),\left(R_{3}, R_{4}\right)$ and $\left(R_{5}, R_{6}\right)$. However, $R_{c 1}$ has less mean square error in comparison to $R_{c 2}$.

From table 3, it has been observed that the expected cost of the proposed classes of chain type estimators $\left(R_{c 1,} R_{c 2}\right)$ are less than the expected cost of $\hat{R}$ and corresponding estimators $\left(R_{1,} R_{2}\right),\left(R_{3}, R_{4}\right)$ and $\left(R_{5,}, R_{6}\right)$ in case of the specified variance. The expected cost of $R_{c 1}$ is less than the expected cost of $R_{c 2}$. 
Table 3 : Expected cost (in Rs.) of the estimators for the specified variance $V_{0}=0.7521$

\begin{tabular}{|c|c|c|c|c|}
\hline & $e_{1}^{\prime}=R s .1 .50$ & $e_{2}^{\prime}=R s . \quad 0.10$ & $e_{2}=R s .9$ & $e_{3}=R s .38$ \\
\hline Estimators & $k_{o p t}$ & $\begin{array}{c}n_{o p t}^{\prime} \\
\text { (approx.) }\end{array}$ & $\begin{array}{c}n_{\text {opt }} \\
\text { (approx.) }\end{array}$ & $\begin{array}{r}\text { Expected cost } \\
\text { (in Rs.) } \\
\end{array}$ \\
\hline$\hat{R}$ & 2.14 & --- & 30 & 492.13 \\
\hline$R_{1}$ & 2.07 & 60 & 18 & 390.98 \\
\hline$R_{2}$ & 1.31 & 62 & 17 & 418.85 \\
\hline$R_{3}$ & 1.82 & 62 & 15 & 341.99 \\
\hline$R_{4}$ & 1.05 & 63 & 13 & 361.29 \\
\hline$R_{5}$ & 2.07 & 54 & 18 & 385.14 \\
\hline$R_{6}$ & 1.31 & 56 & 17 & 412.81 \\
\hline$R_{c 1}$ & 1.82 & 56 & 15 & 338.09 \\
\hline$R_{c 2}$ & 1.05 & 58 & 13 & 357.27 \\
\hline
\end{tabular}

\section{Conclusion}

Hence, we observe that the proposed classes of chain type estimators $\left(R_{c 1}, R_{c 2}\right)$ are more efficient than the corresponding estimators $\left(R_{1}, R_{2}\right),\left(R_{3}, R_{4}\right)$ and $\left(R_{5}, R_{6}\right)$ for fixed sample sizes $\left(n^{\prime}, n\right)$ and for the fixed cost $C \leq C_{0}$. The proposed classes of chain type estimators $\left(R_{c 1}, R_{c 2}\right)$ have also less cost in comparison to cost incurred for the corresponding estimators $\left(R_{1}, R_{2}\right),\left(R_{3}, R_{4}\right)$ and $\left(R_{5}, R_{6}\right)$ for the specified variance. Hence, the proposed classes of chain type estimators have a large number of estimators as members which have equal efficiency for the fixed cost. Hence members are also having equal cost for specified variance. So, we would like to propose the classes of chain type estimators for $R$ which has several more efficient estimators as members of the classes for fixed sample size $\left(n^{\prime}, n\right)$ and also for the fixed cost $C \leq C_{0}$.

\section{References}

[1] M.H. Hansen, W.N. Hurwitz, The problem of non-response in sample surveys, Journal of American Statistical Association, 41, (1946), 517529.

[2] B.B. Khare, S.K. Pandey, A class of estimators for ratio of two population means using auxiliary character in presence of non - response, Journal of Scientific Research, Banaras Hindu University Varanasi, 50, (2000), 115-124.

[3] B.B. Khare, R.R. Sinha, Estimation of the ratio of two population means using auxiliary character with unknown population mean in presence of no-response, Progress of Mathematics, Banaras Hindu University, 36(1, 2), (2002), 337-348.

[4] B.B. Khare, R.R. Sinha, Estimation of finite population ratio using two phase sampling scheme in the presence of non-response, Aligarh Journal of Statistics, 24, (2004), 43-56.

[5] B.B. Khare, R.R. Sinha, Estimation of the ratio of the two population means using multi-auxiliary characters in the presence of non-response, Statistical Techniques in Life Testing, Reliability, Sampling Theory and Quality Control, (2007), 163-171.

[6] S. Rani Srivastava, B.B. Khare, S.R. Srivastava, On generalized chain estimator for ratio and product of two population means using auxiliary characters, Assam Statistical Review, 2(1), (1988), 21-29.

[7] S. Rani Srivastava, S.R. Srivastava, B.B. Khare, Chain ratio-type estimators for ratio of two population means using auxiliary characters, Communication in Statistics-Theory Methods, 18 (10), (1989), 3917-3926.

[8] B.B. Khare, S. Rani Srivastava, Combined generalized chain estimators for ratio and product of two population means using auxiliary characters, Metron, 56 (3-4), (1998), 109-116.

[9] V.K. Singh, Hari P. Singh, Housila P. Singh, D. Shukla, A general class of chain estimators for ratio and product of two means of a finite population, Communication in Statistics - Theory Methods, 23 (5), (1994), 1341-1355.

[10] M.S. Ahmed, The general class of chain estimators for the ratio of two means using double sampling, Communication in Statistics-Theory Methods, 26(9), (1997), 2247-2254.

[11] B.B. Khare, U. Srivastava, K. Kumar, Chain type estimators for ratio of two population means using auxiliary characters in the presence of non-response. Journal of Scientific Research, Banaras Hindu University, 56, (2012), 183-196.

[12] V.N. Reddy (1978), A study of use of prior knowledge on certain population parameters in estimation, Sankhya B 40, (1978), 29-37.

[13] S.K. Srivastava, H.S. Jhajj, A class of estimators of the population means using multi-auxiliary information, Calcutta Statistical Association Bulletin, 32, (1983), 47-56. 